\title{
Viscoelastic Properties of Polymer Solutions ${ }^{1}$
}

\author{
By John D. Ferry ${ }^{2}$
}

\begin{abstract}
In a concentrated polymer solution, the entanglement of long-chain molecules results in a transient network structure, to which may be attributed certain aspects of the viscoelastic behavior of such a system. A suitable mechanical model for representing this network as a first approximation is a retarded Maxwell element with one spring and two dashpots. Experimental measurements of mechanical properties of polymer solutions may be made either by periodic deformation under conditions where inertia forces can be neglected or by propagation of transverse waves. The data are expressed in terms of frequency-dependent parameters from which can be derived the constants of the corresponding mechanical model. A solution of polystyrene in xylene is cited as an example. In this case, analysis in terms of a recent theory of Kuhn suggests that elastic energy may be stored in the network strands by twist against the potential hindering free rotation about bonds in the chains.
\end{abstract}

\section{Entanglement in Concentrated Solutions}

The polymer solutions discussed in this paper lie in the concentration range from 5 to 50 percent-more concentrated than the very dilute solutions commonly used for measurements of viscosity and osmotic pressure and other properties from which the behavior of single molecules is deduced, but more dilute than the usual commercial plastic, which may contain from 50 to 100 percent of polymer mixed with a plasticizer. Solutions in this range are used in plastics technology -in spinning, extrusion, and coating processes. Aside from their technical importance, they are of interest because of their remarkable mechanical, optical, and dielectric properties. Their mechanical properties are intermediate between those of solids and liquids; these solutions are both viscous and elastic.

Examples may be cited to illustrate three very different types. Lightly vulcanized rubber swollen in cyclohexane to a concentration of 20 percent is a quivery, elastic gel. A variety of evidence shows that the long-chain molecules are bound together at widely spaced points by primary chemical bonds, forming a network that can be broken only by chemical decomposition; but there

\footnotetext{
1 This paper was presented as part of the 1946-47 series of lectures on the Properties of High Polymers given at the National Bureau of Standards.

${ }^{2}$ Department of Chemistry, University of Wisconsin.
}

is very little hindrance to motion of the chains except for these occasional cross-links. Polyvinyl chloride dissolved in cyclohexanone at the same concentration is a sluggish gel. There are no primary bonds, as shown by the fact that the gel can be dissolved by adding more solvent. Nevertheless, the tendency of polyvinyl chloride molecules to associate, segmentwise, with dipole interaction (as shown by the ready formation of crystalline regions in the solid state $[1]^{3}$ and association in dilute solution [2]), makes plausible the concept that there is a network here, also, held together by secondary bonds caused by association of the long chains here and there along part of their length. The expectation that association can occur anywhere along a molecule would explain why the gel is sluggish and viscous, in contrast to swollen rubber where the chains are tied only at a few points. It also would explain why a broken gel can "heal" upon standing, especially if warmed; the associations simply form again, the rearrangements being facilitated by heating and cooling the system. Finally, in polystyrene dissolved in xylene at the same concentration of 20 percent, a viscous liquid, there are evidently not even any secondary bonds between chains, since the liquid flows with no yield value. Nevertheless, for very sudden stress, or

\footnotetext{
${ }^{3}$ Figures in brackets indicate the literature references at the end of this paper.
} 
alternating stress, the system behaves as a solid. Such behavior may be at least partly ascribed to entanglement of the long molecules with each other at this concentration. Consideration of the average volume pervaded by a single molecule [3] leads to the conclusion that, for a molecular weight of 100,000 to 200,000 , entanglement, or overlapping of the regions that different molecules on the average pervade, occurs at concentrations as low as 2 to 5 percent. Thus the entanglement at a concentration of 20 percent must be considerable. The resulting structure probably behaves as a network, providing it is deformed quickly enough, before the molecules can move out of the way of each other. A schematic picture contrasting the structures of these three solutions is shown in figure 1.
We are concerned here with attempts to obtain information from measurements of viscoelasticity about this entanglement, which is common to all three types of polymer solutions, and we seek it primarily in solutions like that illustrated in the third part of figure 1, where primary and secondary bonds are absent.

\section{Choice of a Mechanical Model}

It has been customary to represent viscoelastic behavior by models of elastic elements (springs) combined with viscous elements (dashpots, or pistons moving in a viscous medium). It is usually assumed that the viscous elements are Newtonian - the rate of displacement being proportional to the force applied - and that the elastic elements follow Hooke's Law - the displacement
Primary Bonds

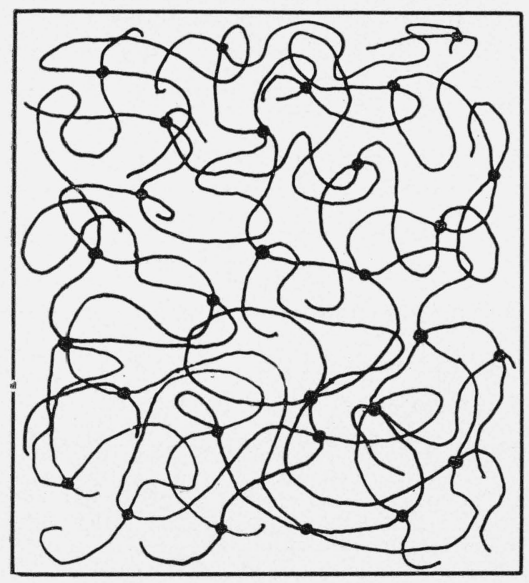

Secondary Bonds

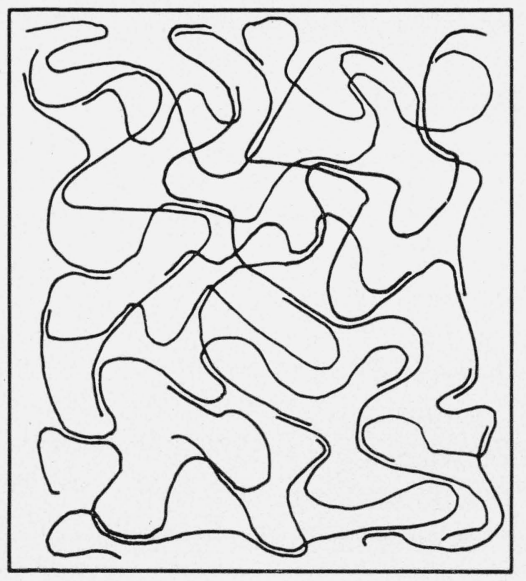

Entanglement

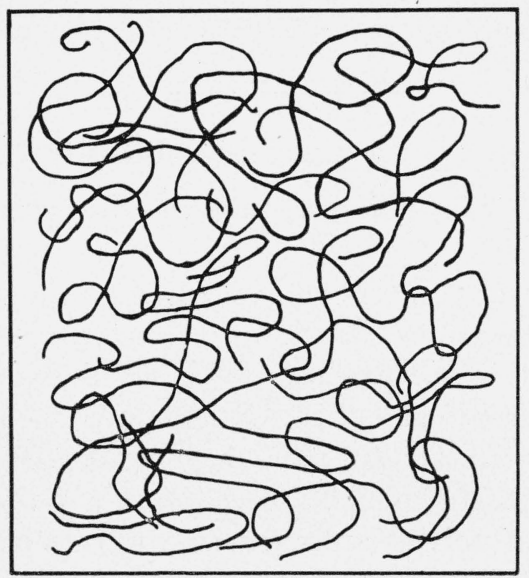

FIGURE 1. Schematic illustration of types of networks in concentrated polymer solutions, involving primary bonds, secondary bonds, and entanglement.

Of course, entanglement must exist to some extent in the first two types of solutions as well as the third [4]. Under certain conditions, such as subjection to stress over comparatively long time periods, which allow opportunity for disentanglement, the effects of transitory overlapping of chains can no doubt be disregarded in comparison with those of the more permanent links represented by primary and secondary bonds. However, for a complete description, entanglement must be taken into account in all three systems. It should be of most significance in experiments where a solution is subjected to very brief, small stresses that do not perceptibly distort the network. being proportional to the force applied. Eyring and his collaborators [5] have shown that in solids, especially fibers, nonlinear elements are necessary to describe mechanical behavior; but in rather fluid solutions, when the applied stresses are small, linear springs and dashpots may be expected to suffice.

If we can imitate the mechanical properties of a certain polymer solution by one particular mechanical model, we can also imitate it by many other mechanical models. This fact was illustrated by Simha a few years ago [6] and was also emphasized in a recent paper by Alfrey and Doty [7]. For example, to represent the behavior of a polymer solution, a Maxwell model may be employed. 
The Maxwell element is a spring and a dashpot in series, and the model employs a number of such elements in parallel. But if such a Maxwell model works, then so also will a Voigt model. The Voigt element is a spring and dashpot in parallel, and the model employs a number of such elements in series. By proper choice of the spring and dashpot constants, these two models will behave exactly the same under all manner of conditions - steady stress, abrupt stress, and oscillating stress.

Since we shall have a choice of mechanical models to represent the viscoelastic behavior of a high polymer solution, it will be advantageous to select one that can be interpreted, as far as possible, in terms of the polymer molecules and the motions that they undergo when the solution is deformed, and in which the elastic and viscous elements may perhaps be identified with molecular processes. The simplest possible model for the third network of figure 1 is a spring in parallel with a viscosity that delays its response to stress and in series with another viscosity that allows relaxation; this may be called the retarded Maxwell element (fig. 2).

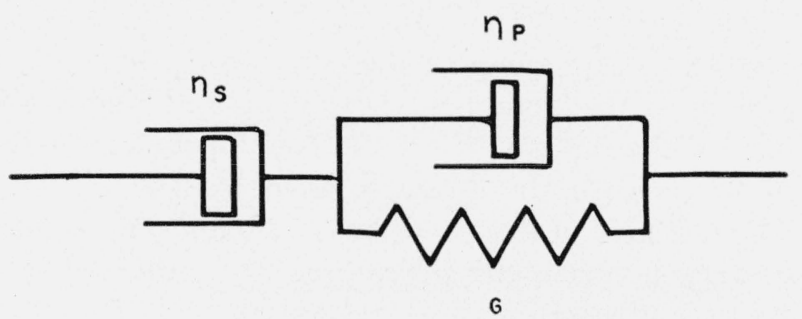

Figure 2. Retarded Maxwell element.

The mechanical properties of some polymer solutions as revealed in certain types of experimental measurements can be represented by this model as a first approximation. It is certain that a more complicated model will be required to represent all mechanical properties. Nevertheless, most of this discussion will be based on the behavior of the simple retarded Maxwell element.

It is natural to identify the spring of this mechanical representation with the average strand of the network between two temporary points of entanglement. There are three possible ways in which such a molecular strand might store elastic energy. (a) Energy is stored by bond deformation (stretching and bending); Kuhn [8] has calculated that the contributions of stretching and bending to elongation of a fully extended paraffin chain should be roughly equal and that the force constant should be proportional to $1 / Z$, where $Z$ is the number of atoms in the chain. For $Z=100$ it should be about 2,000 dyne $/ \mathrm{cm}$. (b) Energy is stored by twist around the bonds against the hindering potential, which interferes with free rotation; Kuhn [9] has calculated that the force constant should be proportional to $1 / Z^{2}$. For the above chain in randomly kinked configuration it should be about 0.5 dyne $/ \mathrm{cm}$. (c) Energy is stored by decrease in entropy when extension restricts the chain to less probable configurations; the force constant is proportional to $1 / Z$ [8], and for the above chain it is about $0.6 \mathrm{dyne} / \mathrm{cm}$.

Mechanisms (b) and (c) are mutually exclusive; if the interval of an experiment is too short to allow rotation over potential barriers into new positions of minimum potential energy, there can be no statistical restoring force or "rubberlike" elasticity; if the interval is long enough to allow many rotational rearrangements, there can be no storage of elastic energy against the hindering potential. The critical time interval is, according to Kuhn, proportional to $Z$. The extension caused by mechanism (a) is probably so small in solutions that it can be neglected altogether. This mechanism is analogous to a very stiff spring in series with our mechanical model, which is never perceptibly stretched by the forces applied.

The two viscous components in the model can also be interpreted roughly. The parallel viscosity is the frictional resistance to rearrangements of chain segments involved in extending the spring, due to both the viscosity of the solvent and the interference of neighboring chains. The series viscosity permits relaxation of stress, due to three possible processes: (a) rotation around bonds into new positions of minimum potential energy, (b) slippage of strand ends by disentanglement, (c) chemical decomposition resulting in breaking of strands. The last of these is ordinarily a very slow process, which may of course be ignored in entanglement networks; the times involved are of the order of days or months. In the case of rubbers, the chemical chain scission has been carefully studied by Tobolsky [10].

If a polymer solution is subjected to stresses that are large enough or prolonged enough in time to produce a substantial distortion of the network from its normal configuration, it may be expected that the mechanical properties will appear quite 
different from those measured under conditions where only slight rearrangements of chain segments are involved. This discussion is concerned only with the latter case - the response of a solution to small, brief stresses. Although such response can be obtained by transient methods, in which a momentary stress is suddenly applied and the resulting deformation is subsequently followed, it is ordinarily more convenient to study the effects of periodically varying stresses [7, 11].

Experimental measurements of the response of a polymer solution (or other viscoelastic material) to sinusoidally varying stress can be expressed in terms of certain parameters that will be presently defined. By comparing the frequency dependence of these parameters with the calculated behavior of models it is possible to select one-that of fig. 2 or one more complicated if necessary - which will represent the data, and to obtain numerical values of the spring constants and dashpot viscosities, which may be related to the nature of the network structure and the properties of the individual network strands.

The methods available for measurements of this sort fall generally into two classes: those in which the inertia of the material can be neglected in the equations of motion, and those in which it cannot [11]. In the former, the size of the sample is considerably less than the wave-length of elastic vibrations, or else the damping characteristics of the material are so marked that no vibrations are propagated. In the latter, the size of the sample is of the same order of magnitude as, or greater than, the wavelength of elastic vibrations, so that vibrations of one kind or another can be set up and measured. The applicability of each method thus depends on the size and consistency of the sample and the frequency range within which measurements are to be made. One example of each class will be given here: the electrodynamic method of Philippoff [12], in which the inertia is neglected, and the method of propagation of transverse waves in extended media [13], in which it is not.

\section{Experimental Methods in Which In- ertial Effects in the Medium Are Neglected}

When a sample of material is subjected to sinusoidally varying shear stress, it responds with a sinusoidally varying strain, which may be out of phase with the stress (fig. 3). At a single

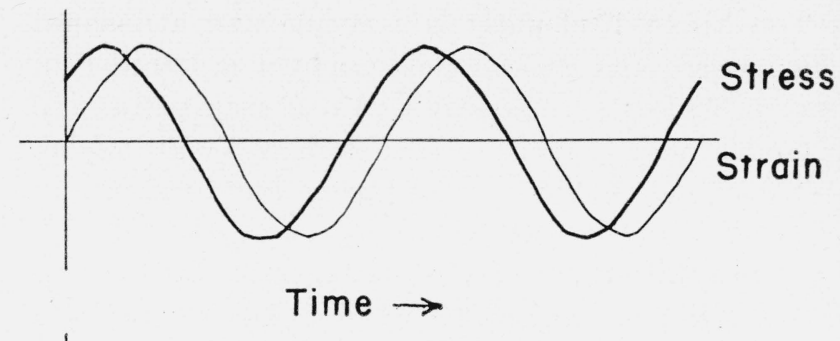

Figure 3. Sinusoidally varying strain in response to sinusoidally varying stress.

frequency, the mechanical characteristics are fully described [11] by two parameters, which may be chosen as $|G|$, the absolute rigidity, or ratio of peak stress to peak strain, and $\psi$, the phase angle between them; or, alternatively, as $G^{\prime}$, the real part of the complex modulus of rigidity, and $\eta^{\prime}$, the real part of the complex viscosity. The relations between these quantities are as follows,

$$
\begin{gathered}
G=G^{\prime}+i G^{\prime \prime} ; n=\eta^{\prime}-i \eta^{\prime \prime} ; \\
G^{\prime \prime}=\omega \eta^{\prime} ; \eta^{\prime \prime}=G^{\prime} / \omega ; \\
|G|=\sqrt{G^{\prime 2}+G^{\prime \prime 2}}=\sqrt{G^{\prime 2}+\omega^{2} \eta^{\prime 2}} \\
\tan \psi=G^{\prime \prime} / G^{\prime}=\eta^{\prime} / \eta^{\prime \prime} .
\end{gathered}
$$

Here $\boldsymbol{G}$ is the complex modulus of rigidity [14] and $\boldsymbol{n}$ is the complex viscosity [15]. The real part of the rigidity, $G^{\prime}$, is the component of stress in phase with the strain divided by the strain. The real part of the viseosity, $\eta^{\prime}$, is the component of stress in phase with the rate of strain divided by the rate of strain.

In describing the behavior of a mechanical model, it is customary to assign each spring a rigidity $G$ and each dashpot a viscosity $\eta$, although dimensions of these constants (dyne $/ \mathrm{cm}^{2}$ and dyne-sec $/ \mathrm{cm}^{2}$ ) are not the same as those of the force constant of a real spring $(\mathrm{dyne} / \mathrm{cm})$ and the viscous resistance of a real dashpot (dyne-sec/cm). A given model will show characteristic frequency dependence of the quantities $G^{\prime}, \eta^{\prime}$, and $|G|$, which may be compared with experimental data. For the retarded Maxwell element (fig. 2), the frequency dependence of $G^{\prime}, \eta^{\prime}$, and $|G|$ is given by the following equations $[15,11]$ :

$$
\begin{gathered}
G^{\prime}=G \omega^{2} \tau_{S}^{2} /\left[1+\omega^{2}\left(\tau_{S}+\tau_{P}\right)^{2}\right] \\
\eta^{\prime}=\eta_{S}\left[1+\omega^{2} \tau_{P}\left(\tau_{S}+\tau_{P}\right)\right] /\left[1+\omega^{2}\left(\tau_{S}+\tau_{P}\right)^{2}\right] \\
|G|=G \omega \tau_{S}\left(1+\omega^{2} \tau_{P}^{2}\right)^{1 / 2} /\left[1+\omega^{2}\left(\tau_{S}+\tau_{P}\right)^{2}\right]^{1 / 2}
\end{gathered}
$$

where $\omega$ is $2 \pi$ times the frequency, and the charac- 
teristic time constants $\tau_{S}$ and $\tau_{P}$ are defined as $\eta_{S} / G$ and $\eta_{P} / G$, respectively, $\eta_{S}$ and $\eta_{P}$ being the series and parallel viscosities. Plots are shown in figures 4 and 5 ; the abscissa is $\log \omega \tau_{S}$, and curves are drawn for different values of the ratio $\tau_{P} / \tau_{S}$. At low frequencies $\eta^{\prime}=\eta_{S}$ and $|G|=G^{\prime}=0$; the model behaves like a single viscosity. At high frequencies, $\eta^{\prime}$ and $G^{\prime}$ approach constant values, and $|G|$, a measure of the actual "stiffness," increases without limit.

The only extensive data with which these model curves can be compared at present are those of Philippoff for the real part of the viscosity, $\eta^{\prime}$, in solutions of cellulose acetate in dioxane [12]. In his apparatus, a solution is subjected to oscillating

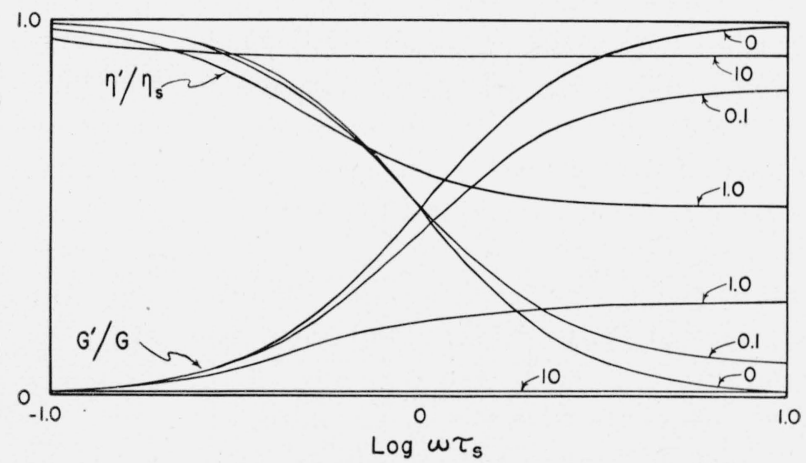

Figure 4. Dependence of $G^{\prime}$ and $\eta^{\prime}$ on frequency for the retarded Maxwell element.

Figures opposite the curves denote values of $\tau_{P} / \tau_{S}$.

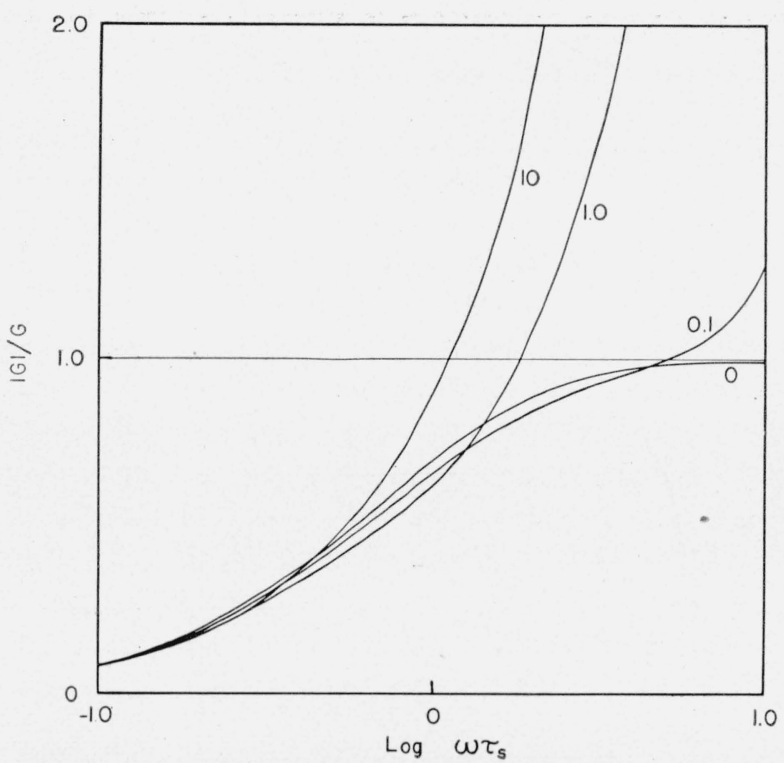

Figure 5. Dependence of $|G|$ on frequency for the retarded Maxwell element

Figures opposite the curves denote values of $\tau_{P} / \tau_{B}$. shear stress by a needle moving up and down along its axis in a narrow tube. The needle is attached to a rather stiff steel band, which is displaced by electromagnets whose field coils are energized by an oscillator and interact in such a way as to provide an electromechanical feedback. Resonance is achieved at various frequencies by adjusting the tension of the band and tuning the feedback circuit. At resonance, $\eta^{\prime}=|f| / a \omega|x|$, where $|f|$ is the maximum force acting (determined by the current drawn, together with a direct-current force-current calibration), $a$ is a constant with dimensions of length involving the depth of immersion and the radii of tube and needle, and $|x|$ is the maximum excursion, determined by a micrometer screw.

Values of $\eta^{\prime}$ obtained by Philippoff for solutions of cellulose acetate in dioxane are plotted against the frequency in figure 6 , and the ratio of $\eta^{\prime}$ to $\eta$, the viscosity in steady flow, is plotted against the logarithm of the frequency in figure 7 . It appears to be approaching zero with increasing frequency, as in the case of the retarded Maxwell element with $\tau_{P}=0$ (the ordinary Maxwell element); thus the retarding viscosity appears to be negligible, at least at the higher frequencies. However, the decrease in $\eta^{\prime}$ is more gradual than for the model, so that the latter must be modified somewhat to describe the results. Philippoff obtained an excellent fit with the relation $\eta^{\prime}=B /\left[1+(\omega \tau)^{3 / 4}\right]$, where $B$ and $\tau$ are empirical constants of uncertain significance; $B$ was not equal to $\eta$, although of the same order of magnitude. Alternatively, a fairly $\operatorname{good}$ fit for $\eta^{\prime} / \eta$ as a function of $\omega \tau_{m}$, where $\tau_{m}$ is the reciprocal of the value of $\omega$ at which $\eta^{\prime} / \eta=1 / 2$, is achieved by the Wiechert-Wagner distribution of relaxation times [16], with $b$, the distribution parameter, chosen as 0.5. Choice of the best modification of the model of figure 2 for describing these results should be facilitated by further experimental work on this and other systems.

A possible method for measuring $\eta^{\prime}$, and under some conditions $G^{\prime}$ as well, for polymer solutions is based on the use of an electromechanical transducer $[17,18]$. When a needle is oscillated along its length, as in Philippoff's apparatus, by a moving-coil loudspeaker, the elastic and viscous characteristics of the mechanical part of the system can be calculated from measurements of changes in resistance and reactance of the coil. This technique has the advantage that a very low energy input suffices for measuring the electrical 


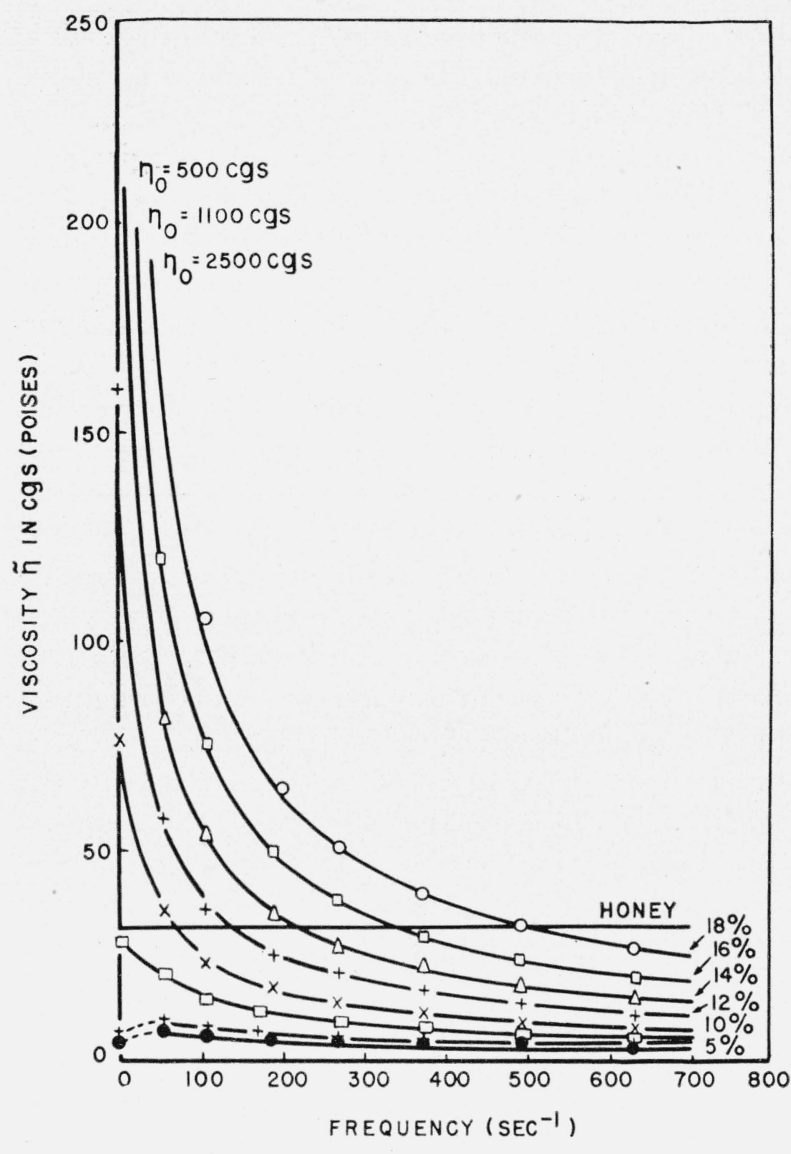

Figure 6. Values of $\eta^{\prime}$ for solutions of cellulose acetate in dioxane, plotted against the frequency (from Philippoff [12]).

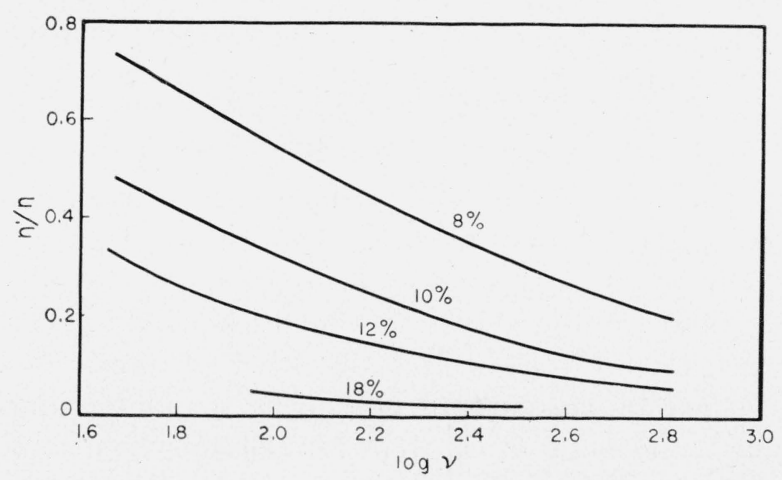

Figure 7. Values of $\eta^{\prime} \eta$ for solutions of cellulose acetate in dioxane, plotted against the logarithm of the frequency (data from Philippoff [12]).

quantities by an impedance bridge, and the amplitude of motion is therefore extremely small (undetectable by ordinary methods), so that the possibility of any non-Newtonian effects is minimized.

\section{Experimental Methods in which In- ertial Effects in the Medium Are Not Neglected: Propagation of Waves}

The simplest case of a mechanical disturbance that involves the inertia as well as the rigidity and viscosity of the material concerned is the propagation of a plane wave of shear in a medium of infinite extent. If the wave is sinusoidal and exponentially damped, it is described by the equation

$$
u=u_{0} e^{i(\omega t-2 \pi x / \lambda)-x / x_{0}},
$$

where $u$ is the displacement, $t$ the time, $x$ the distance from the source of vibration, $\lambda$ the wavelength, and $x_{0}$ the critical damping distance (within which the amplitude falls off to $1 / e$ of its initial value) (fig. 8). For a perfect elastic solid (in which $x_{0}$ is infinite) the modulus of rigidity, $G$, is given by $\omega^{2} \lambda^{2} \rho / 4 \pi^{2}$, where $\rho$ is the density. It is convenient to define for a polymer solution, or other viscoelastic medium, an analogous quantity $\tilde{G}=\omega^{2} \lambda^{2} \rho / 4 \pi^{2}$. The behavior of the system at any given frequency can then be characterized by $\widetilde{G}$, and the ratio $\lambda / x_{0}$ (which is a measure of the severity of the damping).

The two frequency-dependent parameters $\tilde{G}$ and $\lambda / x_{0}$ determined from wave propagation can be used interchangeably with the two parameters $G^{\prime}$ and $\eta^{\prime}$ determined from experiments that do not involve the inertia of the material. They are related by the following equations:

$$
\begin{aligned}
& G^{\prime}=\widetilde{G} \frac{4 \pi^{2}\left[4 \pi^{2}-\left(\lambda / x_{0}\right)^{2}\right]}{\left[4 \pi^{2}+\left(\lambda / x_{0}\right)^{2}\right]^{2}} \\
& \eta^{\prime}=\frac{\widetilde{G}}{\omega} \frac{16 \pi^{3} \lambda / x_{0}}{\left[4 \pi^{2}+\left(\lambda / x_{0}\right)^{2}\right]^{2}} .
\end{aligned}
$$

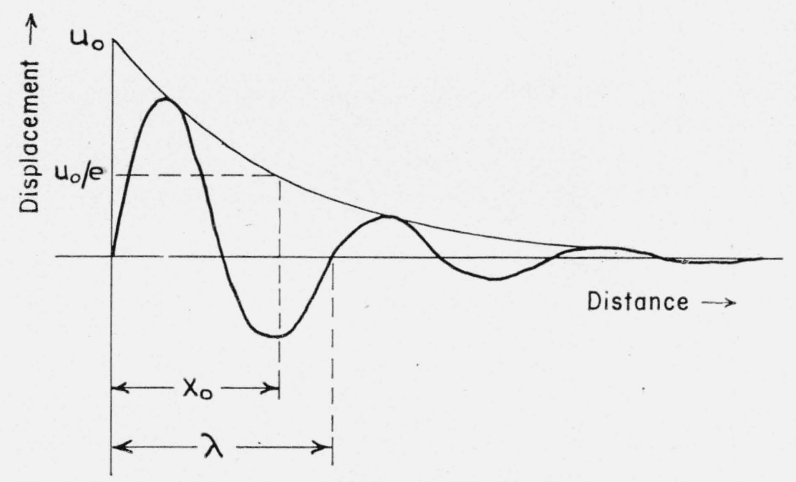

FiguRE 8. Exponentially damped sinusoidal wave. 
It is useful to calculate the frequency dependence of $\widetilde{G}$ and $\lambda / x_{0}$ for mechanical models, for direct comparison with experimental data. For the retarded Maxwell element (fig. 2), the behavior is as follows [11]:

$$
\begin{gathered}
\tilde{G}=G \frac{2 \omega \tau_{S}}{1+\omega^{2}\left(\tau_{P}+\tau_{S}\right)^{2}} \\
\frac{\omega^{2} \tau_{S}{ }^{2}+\left[1+\omega^{2} \tau_{P}\left(\tau_{P}+\tau_{S}\right)\right]^{2}}{\omega \tau_{S}+\sqrt{\omega^{2} \tau_{s}^{2}+\left[1+\omega^{2} \tau_{P}\left(\tau_{P}+\tau_{S}\right)\right]^{2}}} \\
\lambda / x_{0}=\frac{2 \pi\left[1+\omega^{2} \tau_{P}\left(\tau_{P}+\tau_{S}\right)\right]}{\omega \tau_{S}+\sqrt{\omega^{2} \tau_{S}^{2}+\left[1+\omega^{2} \tau_{P}\left(\tau_{P}+\tau_{S}\right)\right]^{2}}} .
\end{gathered}
$$

Plots are shown in figures 9 and 10. Damping is severe at low frequencies because of the series viscosity and at high frequencies because of the parallel viscosity. Only at intermediate frequencies is the damping small enough to permit measurement of wave propagation (for practical purposes, when $\lambda / x_{0}$ is less than 3 or 4$)$. The larger the ratio $\tau_{P} / \tau_{S}$, the smaller is the frequency range within which waves can be observed.

Shear waves may be set up in a solution contained in a rectangular cell by driving a thin plate up and down in its own plane with a loudspeaker [13]. If the cell is long enough in the direction of wave propagation so that the wave is damped

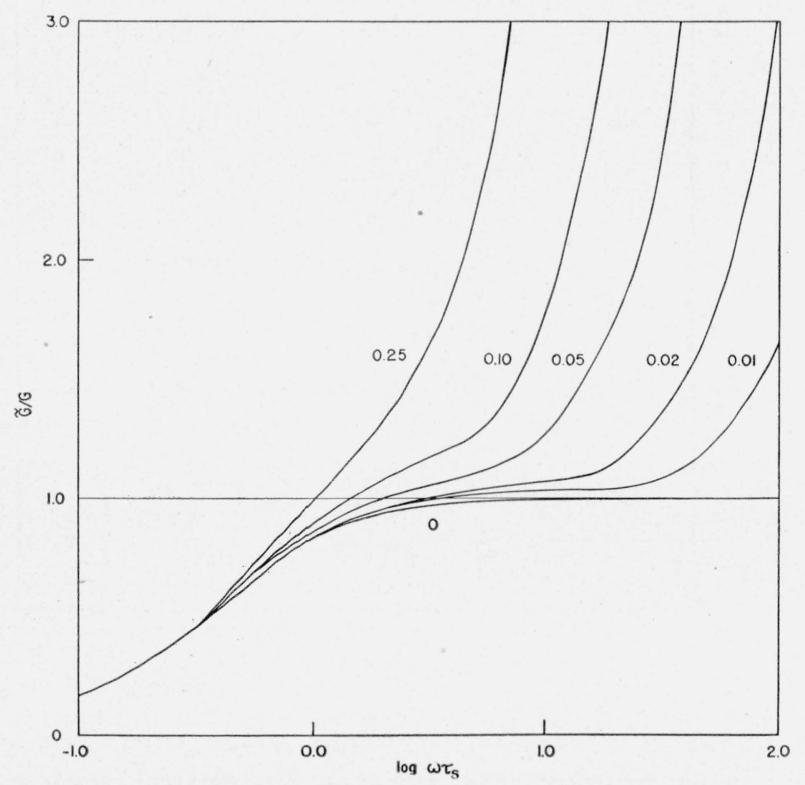

FIgURE 9. Dependence of $\tilde{G}$ on freguency for the retarded Maxwell element.

Figures opposite the curves denote values of $\tau_{P} / \tau_{S}$.

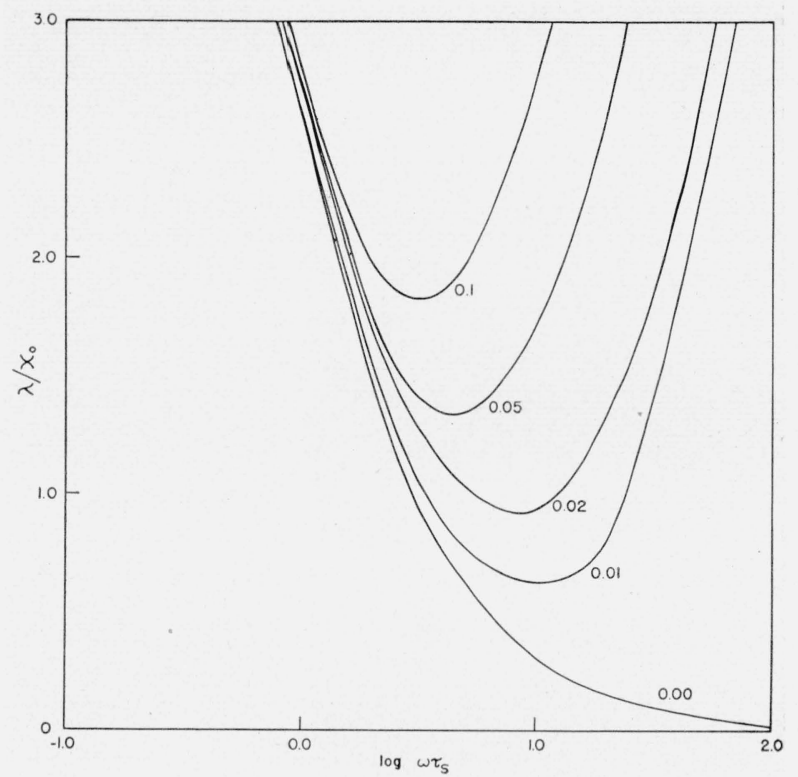

Figure 10. Dependence of $\lambda / x_{0}$ on frequency for the retarded Maxwell element.

Figures opposite the curves denote values of $\tau_{P} / \tau_{S}$.

out before it reaches the end, no reflection occurs, and the medium may be considered of infinite extent in this direction. As for the sides of the cell, through which the wave is observed, a recent investigation [19] shows that their influence on $\tilde{G}$ is negligible but that they do affect the damping somewhat.

If the solution exhibits strain double refraction, the wave can be observed by stroboseopic polarized light flashing at the frequency of vibration. By introducing a double quartz wedge inclined at a small angle to the direction of wave propagation, a pattern is obtained that is closely related to the wave itself (fig. 11), and from which values of $\lambda$ and $x_{0}$ can be calculated. The corresponding values of $\widetilde{G}$ and $\lambda / x_{0}$ (the latter subject to a small correction for the effect of the sides of the cell [19]) are then plotted against the logarithm of the frequency and compared with the curves of figures 9 and 10 , or, if necessary, similar curves for more complicated models.

Wave propagation data for one system, ${ }^{4}$ cellulose xanthate in aqueous sodium hydroxide solutions, follows almost quantitatively the behavior of a retarded Maxwell element. Results on concentrated solutions of polystyrene in xylene [20]

${ }_{4}^{4}$ The author is indebted to the Laboratory of E. I. duPont de Nemours and Co. at Richmond, Virginia, for permission to refer to these results. 


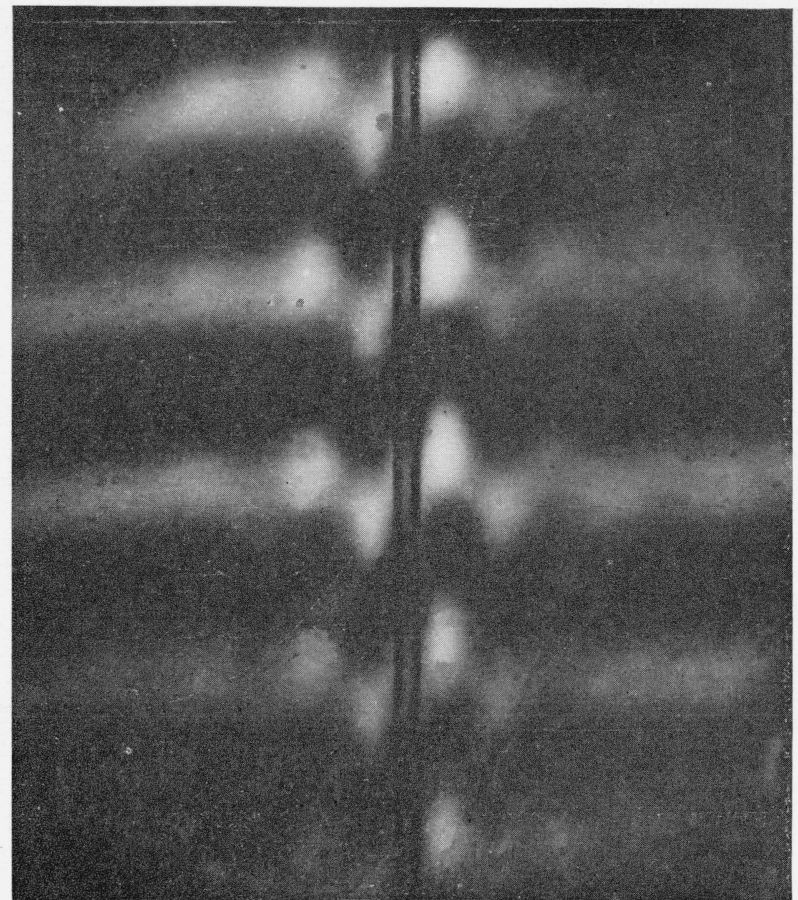

Figure 11. Pattern for transverse waves propagated in 41.5-percent polystyrene in xylene, $15^{\circ} \mathrm{C}, 1,250 \mathrm{c} / \mathrm{s}$.

are in qualitative agreement with the behavior of a retarded Maxwell element with a very small ratio of $\tau_{P} / \tau_{S}$, and within a small frequency range a semiquantitative fit can be obtained (fig. 12). Studies over a wider frequency range are in progress. The rigidity $G$ is found to be proportional to the third power of the concentration.

\section{Interpretation of Model Constants}

From model constants experimentally determined in this way, it should be possible to distinguish among the various molecular mechanisms of elasticity and relation mentioned in section II. The first step is to relate the rigidity of the entanglement network with the elasticity of a single strand.

We define a strand as a segment of a molecule between two points of entanglement, and for a rough calculation assume that, although the strands are randomly kinked, the vector joining the ends of each strand is of uniform length, $l$, and that one-third of these vectors are parallel to each of the $x, y$, and $z$ axes. (Random orientation should affect the result only by a small numerical factor.) If a $y z$-plane cuts $\nu$ strands per square centimeter running in the $x$ direction, then the modulus of elasticity is $E=\nu \kappa l$, where $\kappa$ is the spring constant (force per unit extension) of a single strand. Further, $\nu=N_{s} l / 3$, where $N_{s}$ is the number of strands per cubic centimeter, one-third of which run in the $x$ direction [10], and $G=E / 3$ (Poisson's ratio being negligibly different from $1 / 2$ ), so $G={ }_{\kappa} N_{s} l^{2} / 9$.

We define a point of entanglement as occurring when two monomer units occupy neighboring pseudolattice cells (in the sense used in the theory of thermodynamic properties of polymer solutions [21]), and the four chain segments attached to these two units are suitably oriented to support stress (fig. 13). Each monomer unit has $z$ neighboring cells, where $z$ is the coordination number of the pseudolattice; of these, twe are always occupied by adjacent monomer units in the same chain, the molecular length being considered so great that the chain ends can be neglected. Assuming that the concentration of monomer units in the remaining cells is the same as in the solution as a whole, the number of points of entanglement per cubic centimeter is $N_{c}=p_{e^{-}}$ $(z-2) v(N Z)^{2}$, where $N$ is the number of polymer molecules per cubic centimeter, $Z$ the degree of polymerization, and $v$ the volume of a monomer unit, and $p_{e}$ expresses the probability of orientation for entanglement. The value of $p_{e}$ may be

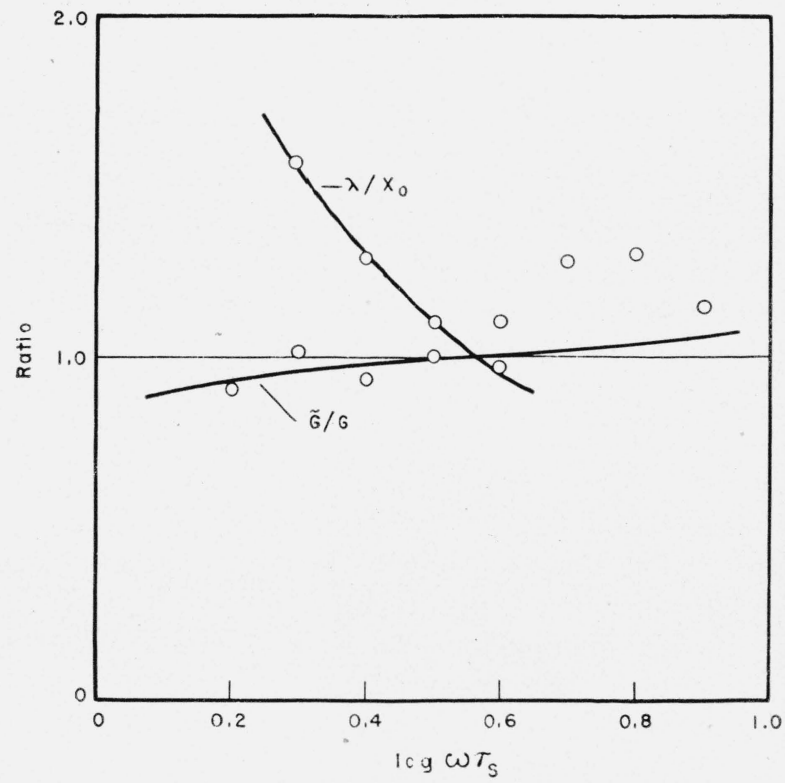

Figure 12. Dependence of $\tilde{G}$ (open circles) and $\lambda / x_{0}$ (filled circles) on frequency for a solution of polystyrene in xylene, concentration $0.39 \mathrm{~g} / \mathrm{cm}^{3}$; temperature $21.6^{\circ} \mathrm{C}$.

Curves are drawn from equations 5 , with $G=1.64 \times 10^{5}$ dyne $/ \mathrm{cm}^{2}, \tau S=$ $4.0 \times 10^{-4} \mathrm{sec}$, and $\tau_{P} / \tau_{S}=0.016$. 


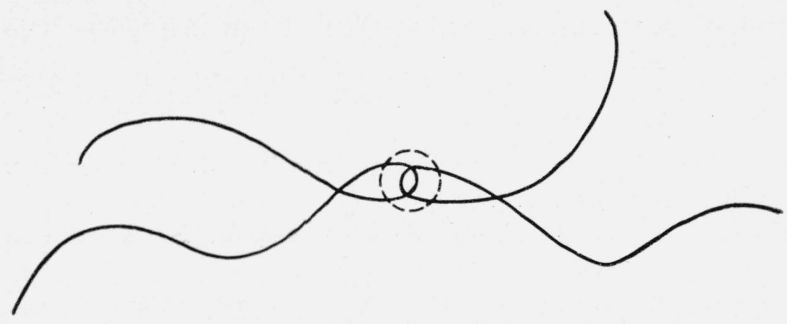

FIGURE 13. Illustration of a point of entanglement.

expected to be smaller than $1 / 4$, and it may be considerably smaller.

If the number of entanglement points per chain is large, $N_{s}=2 N_{c}$. Thus $G=2 p_{e}(z-2) v(N Z)^{2}-$ $\kappa l^{2} / 9$. Now $\kappa$ depends on the strand length, in a manner determined by the elasticity mechanism. Although a previous discussion [20] cited bond bending as a possible mechanism here, it now seems likely that this deformation would be too small to be detectable, and that the choice is between the other two mechanisms listed above. If the strand elasticity is due to twist against the potential hindering free rotation, $\kappa$ should be proportional to $1 / Z_{s}{ }^{2}$, according to Kuhn [9], while if it is a rubberlike elasticity, $\kappa$ should be proportional to $1 / Z_{s}$, where $Z_{s}$ is the number of monomer units per strand. The value of $l^{2}$ is a function of $Z_{s}$; for a randomly kinked chain, $l^{2}=A_{m} b Z_{s}$, where $A_{m}$ and $b$ are lengths related to the dimensions of the monomer unit and the chain flexibility [9]. The product ${ }_{\kappa} l^{2}$ should then be independent of $Z_{s}$ in the case of rubberlike elasticity and inversely proportional in the case of hindered rotation elasticity. Since $Z_{s}=N Z / N_{s}=1 / 2 p_{e}(z-2) v N Z$, and $N Z$ is a measure of the weight concentration, it follows that for rubberlike elasticity the rigidity should be proportional to the square of the concentration and for hindered rotation elasticity it should be proportional to the third power. The observed concentration dependence in polystyrene-xylene solutions corresponds to the latter mechanism.

If the spring represents hindered rotation elasticity, the series dashpot may represent relaxation by rotation over the energy barrier, also treated by Kuhn [9]. The relaxation time for this process is proportional to $Z_{s}$ and hence inversely proportional to concentration. The value of $\tau_{S}$ derived from damping measurements [20] does decrease with increasing concentration in the polystyrene-xylene case, although the data avail- able at present do not permit a test of inverse proportionality.

It is of interest to make a more detailed calculation based on the equation of Kuhn. Kuhn's. expression for $\kappa$ is $192 q / \varphi_{\mu}^{2} j_{\mu} A_{m} b Z_{s}^{2}$, where $q$ is the potential barrier, $\varphi_{\mu}$ the angle between successive positions of minimum energy (here $2 \pi / 3$ ), and $j_{\mu}$ the number of chain bonds per monomer unit (here 2). Substituting this into the above expression for $G$, and noting that $v=M_{0} / N_{0} \rho_{2}=c / \rho_{2} N Z$, where $M_{0}$ is the molecular weight of a monomer unit (here 104), $N_{0}$ Avogadro's number, $\rho_{2}$ the density of the polymer (here 1.05), and $c$ the concentration in grams per cubic centimeter, we obtain

$$
G=\left[(256 / 3) p_{e}^{2}(z-2) N_{0} q / \rho_{2}^{2} M_{0} \varphi_{\mu}{ }^{2} j_{\mu}\right] c^{3} .
$$

For $N_{0} q$ we choose a value estimated by Kuhn from viscosity and flow double refraction of dilute solutions of polystyrene in cyclohexanone, 11,400 cal, and set $z=6$. Then substitution of the experimental value of $G$ for the polystyrene solution described in the preceding section determines the unknown probability $p_{e}$ as 0.0020 . According to this rather remarkably small value, only one pair of monomers in five hundred that occupy neighboring cells in the pseudolattice become entangled. The average number of monomer units in a network strand $\left(Z_{s}\right)$ at the concentration of this solution, $0.39 \mathrm{~g} / \mathrm{cm}^{3}$, is then about 160 .

Further work will be necessary to distinguish the possible roles of intramolecular rotation and slippage at the points of entanglement in stress. relaxation. In any case, it appears that the very low value of the probability factor $p_{e}$ may represent the necessity of rather tight kinking at each point of entanglement to permit support of stress: over the time intervals involved in these experiments.

\section{Summary}

Dynamic studies of the mechanical properties: of concentrated polymer solutions may be applied to gain information concerning molecular entanglement, especially in cases where linear molecules are intertwined with no junctions by primary or secondary bonds among them. Frequencydependent parameters obtained from direct measurements of response to sinusoidal stress, or from the propagation of transverse waves, are compared with the calculated behavior of mechan- 
ical models. In an example cited, a solution of polystyrene in xylene, data within a limited frequency range are fitted by a retarded Maxwell element, characterized by one rigidity and two viscosities. The dependence of the former on concentration suggests that the elastic response involves the hindering potential opposing free rotation about bonds in the molecular chains.

\section{References}

[1] H. Mark, in Burk and Grummitt, The chemistry of large molecules, p. 52. (Interscience Publishers, New York, N. Y., 1943).

[2] P. Doty, H. Wagner, and S. Singer, J. Phys. Chem. 51, 32 (1947).

[3] P. J. Flory, J. Chem. Phys. 13, 453 (1945).

[4] W. Kuhn, J. Polymer Sci. 1, 380 (1946).

[5] A. V. Tobolsky and H. Eyring, J. Chem. Phys. 11, 125 (1943); H. Eyring and G. Halsey, Textile Research J. 16, 13, 284, 335 (1946).

[6] R. Simha, Ann. N. Y. Acad. Sci. 44, 297 (1943).

[7] T. Alfrey and P. Doty, J. Applied Phys. 16, 700 (1945).
[8] W. Kuhn and H. Kuhn, Helv. Chim. Acta, 29, 1095 (1946).

[9] W. Kuhn and H. Kuhn, Helv. Chim. Acta, 29, 609, 830 (1946).

[10] A. V. Tobolsky and R. D. Andrews, J. Chem. Phys. 13, 3 (1945).

[11] J. D. Ferry, W. M. Sawyer, and J. N. Ashworth, J. Polymer Sci. 2, 593 (1947).

[12] W. Philippoff, Physik. Z. 35, 884, 900 (1934).

[13] J. D. Ferry, Rev. Sci. Instruments, 12, 79 (1941).

[14] R. L. Wegel and H. Walther, Physics, 6, 141 (1935).

[15] A. Gemant, Trans. Faraday Soc. 31, 1582 (1935).

[16] W. A. Yager, Physies, 7, 434 (1936).

[17] T. L. Smith, J. D. Ferry, and F. W. Schremp, reported at the High Polymer Division of the American Physical Society, Dec. 30, 1947.

[18] R. D. Fay, J. Acoust. Soc. Am. 15, 32 (1943).

[19] F. T. Adler, W. M. Sawyer, and J. D. Ferry, unpublished work.

[20] J. D. Ferry, J. Am. Chem. Soc. 64, 1323, 1330 (1942); Ann. N. Y. Acad. Sci. 44, 313 (1943).

[21] P. J. Flory, J. Chem. Phys., 10, 51 (1942); M. L. Huggins, J. Am. Chem. Soc. 64, 1712 (1942).

Washington, February 20, 1948. 